Check for updates

Cite this: RSC Adv., 2018, 8, 251

\title{
Detection of mercury ions in water using a membrane-based colorimetric sensor
}

\begin{abstract}
N. A. Azmi, S. H. Ahmad and S. C. Low (D)*
A basic requirement of colorimetric sensors is that they must allow the analyte to access the chromophores. This study elucidates the fundamental sensitivity of membrane morphology (membrane pore sizes between 0.32 and $1.30 \mu \mathrm{m}$ ) and its influence on the loading of chromophores (dithizone, DTZ, in present work), to detect mercury $\left(\mathrm{Hg}^{2+}\right)$ at concentrations ranging from $3 \mathrm{ppm}$ to $10 \mathrm{ppm}$. The flat sheet cellulose acetate (CA) membrane acts as the platform for dithizone loading through non-covalent hydrogen bonding of DTZ to the CA membrane. The membranes were prepared by using different polymer-dissolving solvents (formic acid and acetic acid) with the addition of water as the non-solvent to improve the membrane porosity. A significant positive correlation between the membrane porosity and the sensor's sensitivity was observed through the visible color changes of the colorimetric membrane strip from green (color intensity at $R$-axis of 70 ) to magenta (color intensity at $R$-axis of 187 ) when it was in contact

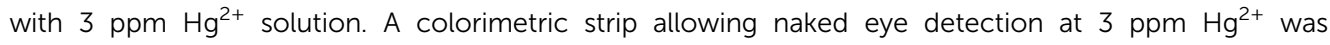
achieved. This work demonstrates that the physical and chemical characteristics of a membrane are important factors that determine the performance and sensitivity of a colorimetric sensor.
\end{abstract}

Received 17th October 2017 Accepted 6th December 2017

DOI: 10.1039/c7ra11450h

rsc.li/rsc-advances fluorescence $\mathbf{2 0}^{\mathbf{2 0} \mathbf{2 4}}$ sensors) appear to offer the simplest technique to determine the presence of target analytes in a testing solution, because of the capacity for naked-eye recognition and short detection time. Indeed, the sensor output signal gives measurable color changes without any spectroscopic instrumentation $^{25}$ upon reaction of a target analyte with the optical indicator ligand that is immobilized on the membrane substrate. ${ }^{26-28}$

The optical indicator diphenylthiocarbazone, also known as dithizone, is a type of chromogenic reagent that serves as a selective and optical transducer for colorimetric sensors. ${ }^{29-31} \mathrm{It}$ contains azo and hydrosulfide groups, which are able to perform as good ligands on the membrane platform. ${ }^{27,32}$ For example, Zargoosh and Babadi ${ }^{33}$ developed an optical sensor by covalently immobilizing dithizone onto an agarose membrane for direct detection of $\mathrm{Pb}^{2+}$ ions in industrial waste and environmental samples without any pre-concentration step. The absorbance differences obtained in their research show the feasibility of using dithizone as an optical indicator for fast metal ion detection. Furthermore, Momidi et al. ${ }^{34}$ proposed the idea of colorimetric chemosensors developed from thiocarbohydrazide derivatives, shedding light on the possibility of selective detection of heavy metal ions in aqueous medium. In their study, ${ }^{34}$ receptor moieties comprising hydroxy groups $(-\mathrm{OH})$, thiocarbonyl groups $(-\mathrm{C}=\mathrm{S}-)$ and imine groups $(\mathrm{C}=\mathrm{N})$ serve as the binding sites for cation detection. For example, the binding of $\mathrm{Hg}^{2+}$ ions to the receptor leads to a visible color change from colorless to yellow and from colorless to pale pink for $\mathrm{Cd}^{2+}$ ions. However, the data collection relies on UV-visible
School of Chemical Engineering, Engineering Campus, Universiti Sains Malaysia, Ser Ampangan, 14300 Nibong Tebal S.P.S. Penang, Malaysia.E-mail: chsclow@usm.my; siewchun@gmail.com; Fax: +60 4599 6908; Tel: +60 45996412 
spectroscopic titration experiments and Benesi-Hildebrand (B$\mathrm{H})$ plots, which require trained personnel and infrastructure. This finding would be more interesting if there was no need for sophisticated devices to observe the color changes.

The dithizone ligand is able to form colored complexes with metal ions, where the response of the color change is dependent on the types of the metal ions that react. Our previous work focused on the conceptual study of a dithizone-colorimetric sensor and its binding mechanism with a cellulose acetate (CA) membrane, which was used as the immobilization and detection platform. ${ }^{35}$ Various metal ions including $\mathrm{Hg}^{2+}, \mathrm{Cu}^{2+}$ and $\mathrm{Zn}^{2+}$ were tested. The study confirmed the feasibility of applying a porous CA membrane as the solid detection platform, and its sensitive binding interactions with dithizone demonstrated its practical applications for on-site quick detection of heavy metals. The prepared dithizone-CA colorimetric sensor exhibited quick detection (within $1 \mathrm{~min}$ ) and was found to be capable of displaying distinctive color changes in response to the presence of $\mathrm{Hg}^{2+}, \mathrm{Cu}^{2+}$ and $\mathrm{Zn}^{2+}$ ions in the testing samples.

Indeed, the effectiveness of a colorimetric sensor is also influenced by the choice of substrate (platform). The most frequently used inorganic substrates, such as glass and silica gel, are dimensionally stable (resistant to swelling). Unfortunately, these inorganic substrates have the disadvantage of limited surface area and porosity. ${ }^{36}$ On the other hand, organic polymer substrates, such as polyvinylidene difluoride $(\mathrm{PVDF})^{37,38}$ and cellulose derivatives, ${ }^{39}$ are commonly used in colorimetric sensors because they offer high mechanical strength, and good thermal and chemical stability. Moreover, they are commercially available with several types of microstructure. Therefore, membrane-based sensors with organic polymer substrates have been extensively explored in recent years. The immobilization capacity of a transducer membrane to bind the optical indicator ligand ultimately determines the feasibility and performance of a sensor. ${ }^{40}$

A polymeric membrane is often used as the porous substrate to hold the optical indicator ligand. Indeed, the porosity of a membrane is critical for the rapid exposure of the chromophores to the testing solutions that contain potential analytes. An effective sensor can be achieved with high-porosity membranes that serve as nano- or micro-structured supports. ${ }^{41}$ Unfortunately, the majority of immobilization strategies for optical indicator ligands have involved conventional practices, which focus on immobilizing procedures and/ or binding interactions of the optical indicator ligand to the substrate used for immobilization. Indeed, in-depth studies of the substrate/membrane used to immobilize chromophores, in terms of the membrane material and pore structure, are also essential. Varying the membrane morphologies will affect the immobilization mechanisms, which will further determine the binding sensitivity of a colorimetric sensor. ${ }^{27,42}$

A basic requirement of all colorimetric sensors is to allow the analyte access to the chromophores. This study elucidates the fundamental sensitivity of the membrane morphology and its influence on the loading of chromophores (dithizone in the present work) to detect $\mathrm{Hg}^{2+}$. The first part of the research was focused on the membrane synthesis by manipulating the membrane pore structures with various polymer-dissolving solvents (formic acid and acetic acid) and non-solvent (deionized water, DI). Then, the membrane-dithizone interactions and the dithizone loading capacity, based on different membrane pore structures, were evaluated in terms of the chemistry interaction properties and the physical morphology of the membrane. The sensitivity of the sensor was determined by the naked-eye detection of $\mathrm{Hg}^{2+}$ at different concentrations in aqueous media and by quantitatively analyzing the membrane dot color intensity using color definition data of RGB triplet color space.

\section{Experimental}

\subsection{Materials}

Cellulose acetate (CA) polymer (with an acetyl content of 40\%) and acetic acid (ACS reagent $\geq 99.7 \%$ ) were supplied by SigmaAldrich (St. Louis, MO). Formic acid (98-100\%), ethanol, diphenylthiocarbazone (dithizone, DTZ) and mercury(II) nitrate monohydrate, were supplied by Merck (Darmstadt, Germany). All chemicals used were of analytical grade and used without further purification.

\subsection{Membrane preparation}

Different microstructures of the membrane platforms were prepared by dissolving a specific concentration of CA polymer in formic acid (FA) and acetic acid (AA) solvent, as per the membrane dope formulations tabulated in Table 1 . The method is essentially the same as that given in our previous paper ${ }^{35,43}$ with some adjustments. First, the polymer dopes were stirred at $250 \mathrm{rpm}$ for 4 hours to ensure the complete dissolution of polymer and then the mixture was degassed to remove air bubbles. Subsequently, the casting dope was cast onto a glass plate with a $500 \mu \mathrm{m}$ clearance gap of the casting blade. The castfilm was then immersed in a distilled water coagulation bath overnight. Then, the solidified membrane was removed from the coagulation bath and immersed in ethanol solution for 4 hours. Lastly, the prepared membrane was air-dried and stored in a sealed plastic bag prior to further use for characterization, functionalization or performance tests.

The surfaces and cross-sectional morphologies of the membranes were observed using a scanning electron microscope (Hitachi TM3000, Tokyo, Japan) at an accelerating voltage

Table 1 Preparation of membranes at different polymer dope compositions

Casting dope composition (wt\%)

\begin{tabular}{lrrrr} 
Membrane id & CA & \multicolumn{1}{c}{ AA } & \multicolumn{1}{c}{ FA } & DI (non-solvent) \\
\hline CA-AA & 17.0 & 83.0 & 0.0 & 0.0 \\
CA-FA & 17.0 & 0.0 & 83.0 & 0.0 \\
CA-FA:30DI & 17.0 & 0.0 & 58.1 & 24.9 \\
CA-FA:40DI & 17.0 & 0.0 & 49.8 & 33.2
\end{tabular}


of $5 \mathrm{kV}$. For cross-sectional analysis, the membranes were fractured using liquid nitrogen. All samples were coated with a layer of platinum (Pt) to prevent surface charge. As for the chemical properties of the membrane, the presence of functional groups with affinity to bind with dithizone was analyzed using a Thermo Scientific FTIR spectroscopy system (NICOLET iS10, USA). Each spectrum was obtained from 32 scans at a $45^{\circ}$ incident angle using a diamond crystal over the wavenumber range of $1800-1100 \mathrm{~cm}^{-1}$.

\subsection{Immobilization of dithizone (DTZ) on the membrane surface}

The flat sheet CA membrane was cut into $5 \mathrm{~mm} \times 40 \mathrm{~mm}$ strips, which were used for dithizone immobilization by immersion in 100 ppm DTZ solution in ethanol for $20 \mathrm{~min}$. Subsequently, the wet membrane was removed and allowed to air dry for 2 hours. The successfully DTZ-coated membrane changed from opaque white to dark green, indicating the successful immobilization of dithizone on the CA membrane surface. The sensor strips were then ready to be used for the trace analysis of mercury ions.

\subsection{Trace analysis of mercury using DTZ-membrane optical sensor}

The qualitative and quantitative responses of the DTZ-coated membrane sensors were studied by dipping the sensor strips in $4 \mathrm{~mL}$ of $\mathrm{Hg}^{2+}$ solutions (ranging from $3 \mathrm{ppm}$ to $10 \mathrm{ppm}$ ) for 16 hours to ensure complete color change in response to the presence of $\mathrm{Hg}^{2+}$ ions. The qualitative assessment of the color change for the membrane strips was performed with the naked eye and the membrane color image was quantitatively converted to the RGB triplet color space. RGB triplet color space is color definition data in which red, green and blue colors are added together in a specific color space to reproduce a broad array of colors. Color images of the membrane sensors $(5 \mathrm{~mm} \times 40$ $\mathrm{mm}$ ) before and after testing with $\mathrm{Hg}^{2+}$ ions were taken using a digital camera. The photo images were divided into 18 portions $(4.5 \mathrm{~mm} \times 2.5 \mathrm{~mm})$ in which the hue saturation value (HSV) and RGB color space of the sensor strips were processed by color assessment software (imagecolorpicker.com). The 3dimensional RGB values from the 18 portions of a single image were then analyzed in a $3 \mathrm{D}$ scatter plot (red, green and blue color space representing the $z$-, $x$ - and $y$-axes), by using an online analytics and data visualization tool, Plotly (Montreal, Quebec, Canada).

\section{Results and discussion}

The efficacy of a colorimetric sensor relies upon the binding interactions between the optical indicator ligand (dithizone, DTZ in present work) and the immobilizing platform (cellulose acetate, CA, membrane) to push the detection ability of the sensor to the lowest possible concentration. Hence, the key structural features of the CA polymer and the formed membranes were verified by ATR-FTIR (Fig. 1) prior to being used as the porous substrate to immobilize DTZ. In Fig. 1, a strong peak observed at around $1735 \mathrm{~cm}^{-1}$ was attributed to the $\mathrm{C}=\mathrm{O}$ stretching vibration of the overlapped ester-carbonyl (acetyl) groups that are connected to the ring structure of the CA polymer. $\mathrm{C}=\mathrm{O}$ is believed to be responsible for binding with DTZ through exposure to the partial negative charge on the carbonyl oxygen atom where the electrons are delocalized between the two oxygen atoms. This will be displayed in more detail later in Fig. 2.

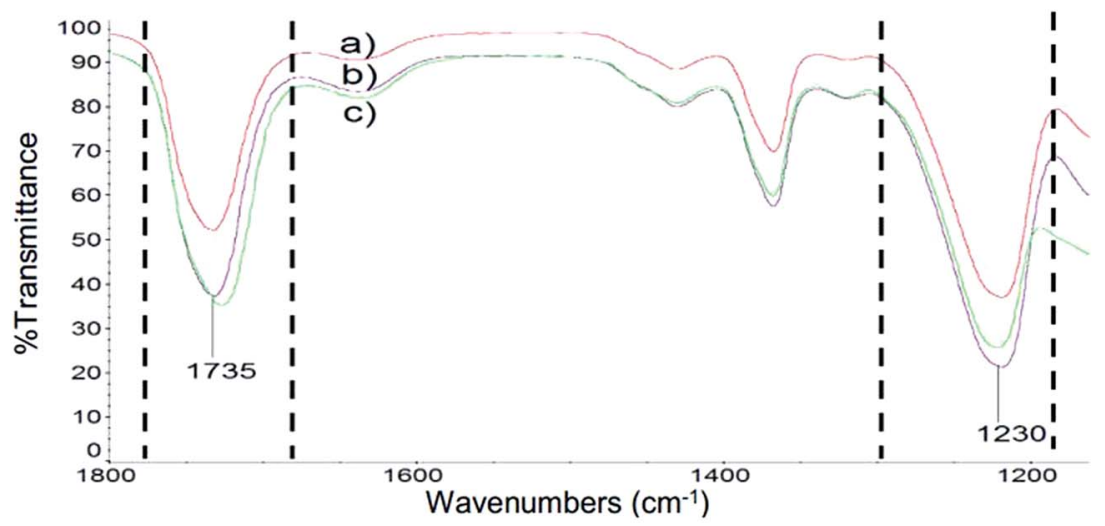

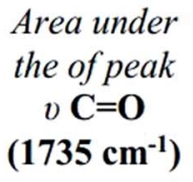

10.61

15.67

17.74

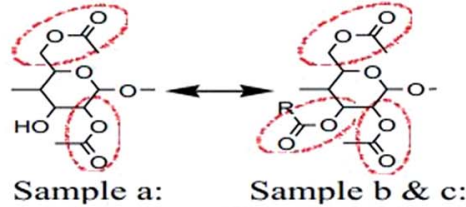

Sample a: CA polymer Sample b: CA-AA membrane Sample c: CA-FA membrane

\section{Area under the of peak $v$ C-O-C $\left(1230 \mathrm{~cm}^{-1}\right)$}

17.37

25.06

22.47

Fig. 1 ATR-FTIR spectra of (a) CA polymer, (b) CA-AA membrane and (c) CA-FA membrane. 


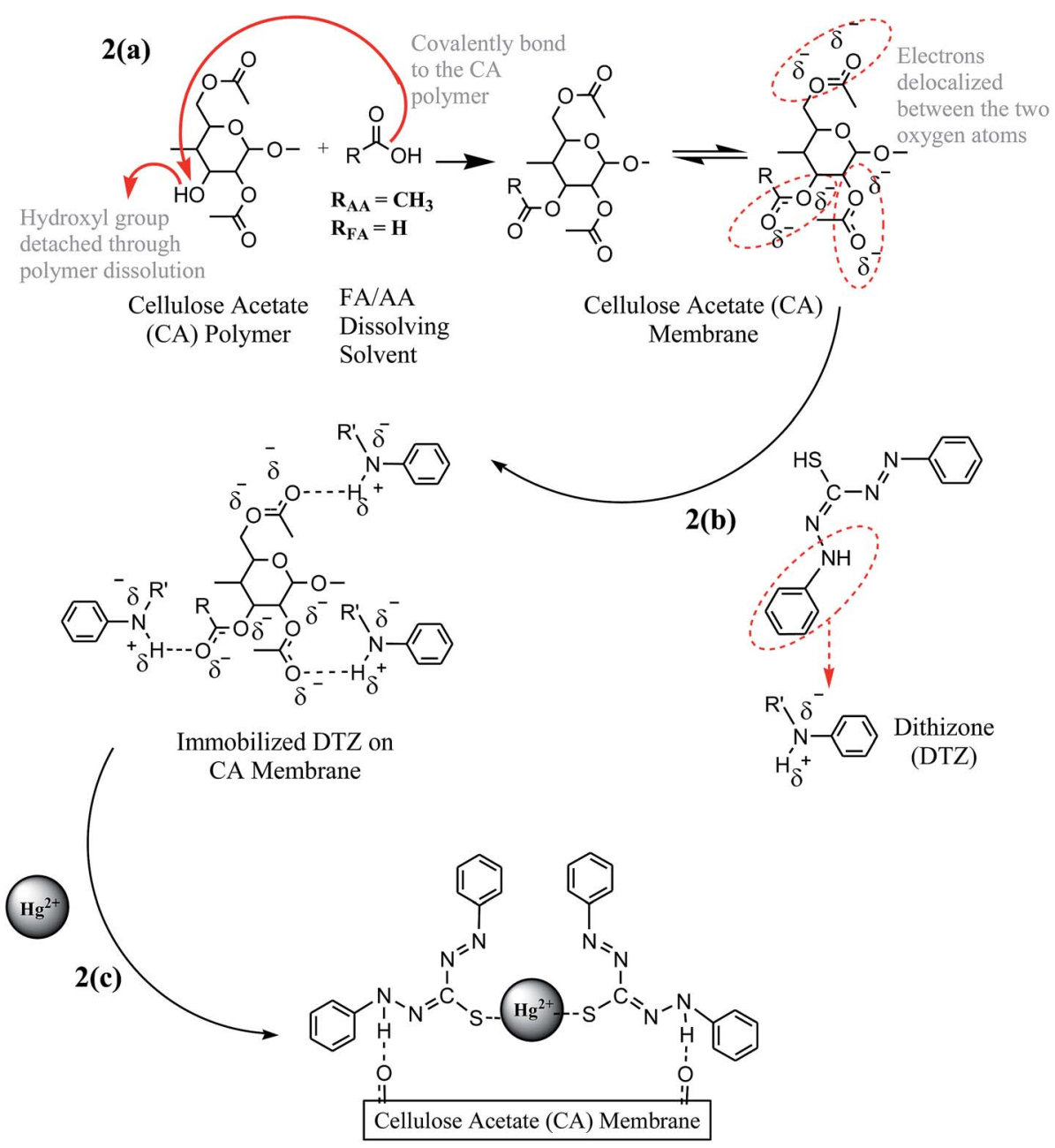

Fig. 2 Schematic representation of (a) CA membrane formation, (b) DTZ interaction with CA membrane and (c) $\mathrm{Hg}^{2+}-\mathrm{DTZ}$ complex.

Interestingly, an increase in the peak intensity of $\mathrm{C}=\mathrm{O}$ was observed after membrane formation. This increase in peak intensity may be due to the interactions between the FA/AA dissolving solvent and the CA polymer during membrane formation, ${ }^{\mathbf{1 6}}$ which is schematically explained in Fig. 2a. On average, the area under the $\mathrm{C}=\mathrm{O}$ peak of the CA-FA membrane (17.74) was found to be slightly higher than that of the CA-AA membrane (15.67) and the CA polymer (10.61), with the peak somewhat shifted from $1735 \mathrm{~cm}^{-1}$ to $1730 \mathrm{~cm}^{-1}$. It is believed that, during hydrolysis, the hydroxyl group connected to the polymer ring structure of CA was broken through polymer dissolution, eventually leaving the central carbon atom to be electropositively charged. Subsequently, the deprotonated carboxyl group from AA or FA solvent was covalently bonded to the carbon atom of the CA polymer (Fig. 2a). Therefore, the peak intensities of $\mathrm{C}=\mathrm{O}$ increased after the membrane formation (Fig. 1).

Similarly, a strong C-O-C stretching peak of ether groups was found at $1230 \mathrm{~cm}^{-1}$ (Fig. 1). At this identified peak, the $\mathrm{C}-\mathrm{O}-\mathrm{C}$ peaks of the CA-AA and CA-FA membranes were obviously stronger than those of the CA polymer, mainly due to dissolution of solvent during the membrane formation, as discussed above.
The CA-AA and CA-FA membranes possess higher areas under the peaks (25.06 and 22.47, respectively) than the CA polymer (17.37), due to the covalent interaction between a -COOR group from the FA/AA solvent and the CA polymer. In this study, the strong carbonyl group of $\mathrm{C}=\mathrm{O}$ with a partially negative charge (Fig. 2a) has a higher electron affinity to attract the partially positive DTZ (Fig. 2b) as compared to the C-O-C ether group. Therefore, it is believed that the carbonyl group acts as the main binding site for DTZ loading. Thus, the latter discussions are focused on the $\mathrm{C}=\mathrm{O}$ functional group of the membrane.

From Fig. 2a, it is fundamental to note that the reaction between the FA/AA solvent and the CA polymer consequently leads to an extra functional peak of $\mathrm{C}=\mathrm{O}$ and this additional functional group is used for DTZ immobilization (Fig. 2b). In addition, the CA membrane is hydrophilic in nature and has a good wettability to polar solvents. This fact has further strengthened our confidence regarding the presence of the more $\mathrm{C}=\mathrm{O}$ polar bond, and chemisorption of DTZ in polar solvent (ethanol) can be easily achieved. Interactions between DTZ and the CA membrane are schematically sketched in Fig. 2b. In this study, DTZ worked as an active optical ligand adsorbed onto the membrane surface for heavy metal detection. 
In order to immobilize dithizone onto the CA membrane, a pair of hydrogen-bond donor and acceptor groups need to be recognized. In dithizone, the electronegative nitrogen atom to which the hydrogen atom is bonded strips electron density from the hydrogen atom $\left(\mathrm{N}^{\delta-} \cdots \mathrm{H}^{\delta+}\right)$, which leads to a partial positive charge on the hydrogen atom. In this case, the partially negative nitrogen from dithizone acts as a hydrogen-bond donor. On the other hand, it was expected that the $\mathrm{C}=\mathrm{O}$ ester-carbonyl (acetyl) group from the CA membrane will become partially negative when the electrons are delocalized between two oxygen atoms (Fig. 2a). Delocalization of the electron pairs is possible since these oxygen atoms possess the same number of unpaired electrons. This partially negative oxygen atom of the carbonyl group acts as a hydrogen-bond acceptor to interact with DTZ. As a possible mechanism, the partially positive hydrogen atom (-NH group) of DTZ is electrostatically attracted to the partially negative oxygen atom (carbonyl group, $\mathrm{C}=\mathrm{O}$ ) of $\mathrm{CA}$ (Fig. 2b). The $\mathrm{H}^{+}$is partially transferred from -NH (hydrogen-bond donor) to the carbonyl oxygen atom (hydrogen-bond acceptor). This non-covalent hydrogen bonding of DTZ to the CA membrane eventually creates a stable binding between the CA polymer and DTZ, which is particularly crucial for an effective membrane sensor to form the $\mathrm{Hg}^{2+}-\mathrm{DTZ}$ complex (Fig. 2c) during the detection of mercury in aqueous solution.
From the above discussion, it is very likely that the CA-FA membrane will be in a good position for DTZ immobilization with its stronger $\mathrm{C}=\mathrm{O}$ peaks, as evidenced in Fig. 1 . However, successful DTZ immobilization does not merely depend on the chemical properties, but is also affected by the physical microstructure of the membrane. In this work, the produced CA-AA and CA-FA membranes were assembled into colorimetric sensors to detect $\mathrm{Hg}^{2+}$ in the concentration range of 3-10 ppm, and the results are depicted in Fig. 3. During the sensor assembly, the neat membranes were first immersed in DTZ solution until the membrane strips changed from opaque white (Fig. 3b) to dark green (Fig. 3c). The retained green color on the membrane strips after several washing steps reaffirmed the successful immobilization of DTZ on the membrane surfaces, which were then ready to be used to detect $\mathrm{Hg}^{2+}$.

Upon immersion of the sensor strips in aqueous $\mathrm{Hg}^{2+}$ at $3 \mathrm{ppm}$, a portion of the membrane strip started to change from green to orange for both the CA-AA-DTZ and CA-FA-DTZ membranes. The color change was due to the formation of the $\mathrm{Hg}^{2+}-\mathrm{DTZ}$ complex in solution as described in Fig. 2c. The $\mathrm{Hg}^{2+}-\mathrm{DTZ}$ complex, with a $\mathrm{Hg}^{2+}$ : DTZ ratio of $1: 2$, was formed through the binding sites of DTZ at nitrogen or sulfur atoms. ${ }^{44-46}$ Apparently, two DTZ atoms are required to form a complexation reaction with $\mathrm{Hg}^{2+}$ ions:

\section{CA-AA Membrane}
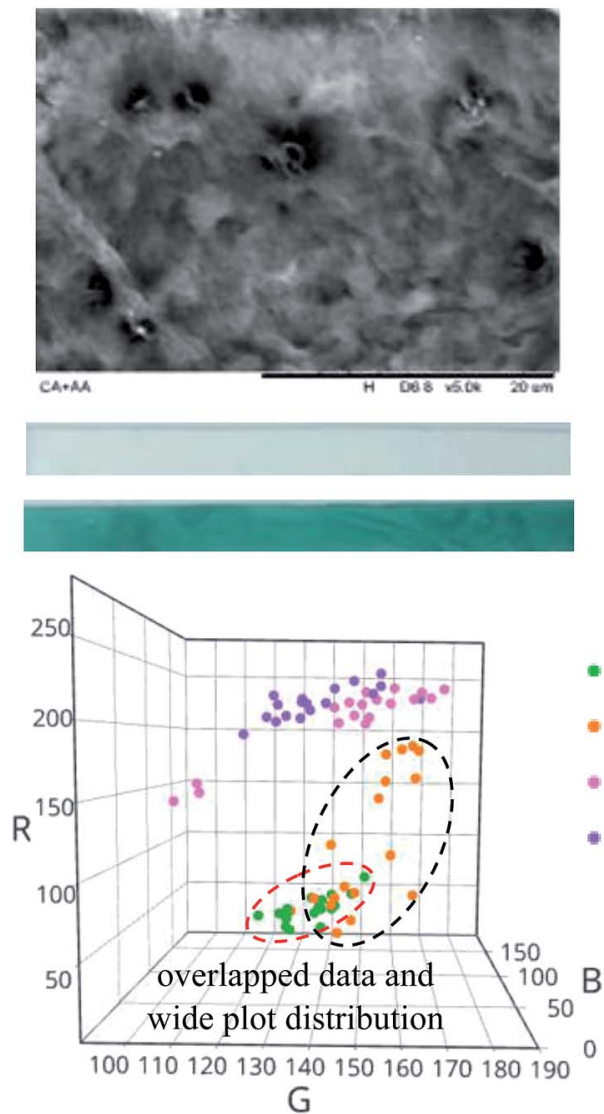

(b)

(c)

(a)
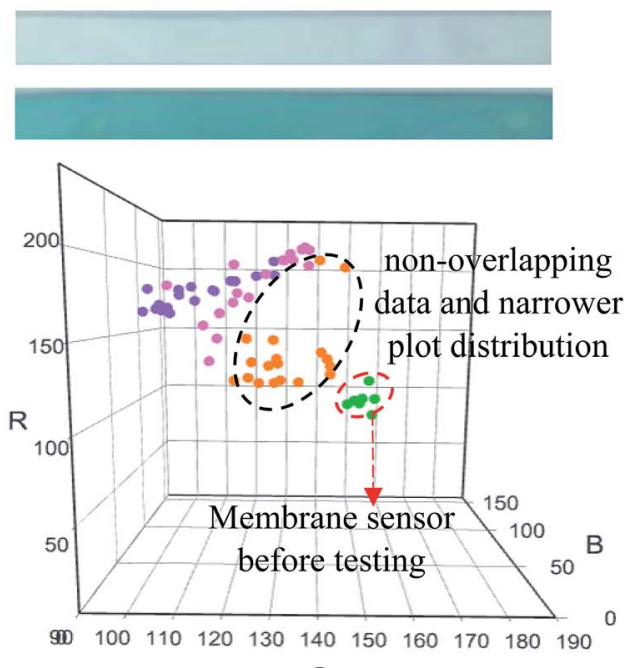

G

Fig. 3 (a) SEM surface morphology of the CA membranes. (b) Opaque white color of CA membranes. (c) Dithizone (DTZ) immobilized on CA membrane strip or the control membrane. (d) Performances of DTZ-colorimetric sensors at different $\mathrm{Hg}^{2+}$ concentrations (3,5 and 10 ppm). 


$$
\begin{gathered}
\mathrm{H}_{2} \mathrm{DTZ}+\mathrm{CA} \text { membrane }=(\mathrm{HDTZ})-\mathrm{CA} \\
\mathrm{Hg}^{2+}+2(\mathrm{HDTZ})-\mathrm{CA}=\mathrm{Hg}^{2+}(\mathrm{DTZ}-\mathrm{CA})_{2}+2 \mathrm{H}^{+}
\end{gathered}
$$

where $\mathrm{H}_{2} \mathrm{DTZ}$ is dithizone.

The hydrogen-bond donors in dithizone $\left(\mathrm{H}_{2} \mathrm{DTZ}\right)$ are nitrogen and sulfur atoms (Fig. 2b). In present work, the $-\mathrm{NH}$ group of DTZ is involved in a hydrogen bonding interaction with the $\mathrm{C}=\mathrm{O}$ group of the CA membrane (eqn (1)), leaving the sulfur atom available to bind with $\mathrm{Hg}^{2+}$ ions (eqn (2)). During hydrolysis in $\mathrm{Hg}^{2+}$ solution, the hydrogen atom from the $-\mathrm{SH}$ group leaves the sulfur atom with one free valence electron. In order to form a stable $\mathrm{Hg}^{2+}$-DTZ complex, two sulfur atoms of the DTZ molecule are required (Fig. 2c), thus contributing to the DTZ $: \mathrm{Hg}^{2+}$ ratio of $2: 1$, as described in eqn (2). Theoretically, the sulfur group has a higher tendency to interact with $\mathrm{Hg}^{2+}$ ions due to its strong thiophilic properties. ${ }^{47}$ According to the Hard and Soft Acids and Bases (HSAB) theory, a soft acid will combine with a soft base and vice versa. Since mercury $\left(\mathrm{Hg}^{2+}\right)$ is a soft acid, it prefers to ligate through the sulfur atom rather than the nitrogen atom.

In this work, the analysis of dot color intensity (RGB triplet value) was used to confirm the extent of the color changes on the membrane sensor. Visual evaluation of the colors was carried out by comparing the dot color intensity of the tested sensors with their control membranes (membrane sensor before testing with the $\mathrm{Hg}^{2+}$ solution). The results are depicted in Fig. 3d. An overlapped color intensity plot was obtained for the CA-AA-DTZ membranes before and after testing with $3 \mathrm{ppm}$ of $\mathrm{Hg}^{2+}$ solution (left 3D scattered plot in Fig. 3d). These overlapped RGB values indicate the insignificant color changes or low efficiency of the CA-AA-DTZ sensor when it was used to detect a low concentration $(3 \mathrm{ppm})$ of the $\mathrm{Hg}^{2+}$ solution. The membrane sensor only showed a weak color change from green to greenish-orange. In contrast, the CA-FA-DTZ sensor demonstrated a non-overlapping data plot (right 3D scattered plot in Fig. 3d), even when it was used to detect $\mathrm{Hg}^{2+}$ at low concentration (3 ppm). A more precise data distribution (lower standard deviation) was found, implying better color change performance with homogeneous color distributions on the surface of the membrane strips.

The correlation between $\mathrm{Hg}^{2+}$ concentration and the effectiveness of the optical sensors (the extent of the color change) was highly related to the difference between the RGB value of the tested sensor and that of its control membrane, as depicted in Fig. 3d. Hypothetically, a DTZ-membrane that captures a higher amount of $\mathrm{Hg}^{2+}$ will demonstrate a color distribution that moves towards to the hot color region (red, $R$-axis, reacted color from $\mathrm{Hg}^{2+}$-DTZ complexes) from the cold color region (green, $G$-axis, the original DTZ-membrane color). It was expected that the greater the color intensity difference of the tested strip (hot color) from the control membrane (cold color) at the $R$-axis, then the higher the detection efficiency of the membrane to capture $\mathrm{Hg}^{2+}$. In this study, the membranes changed from green (control membrane before testing with the $\mathrm{Hg}^{2+}$ ion solution) to orange and magenta (membranes tested with $\mathrm{Hg}^{2+}$ solution with the formation of $\mathrm{Hg}^{2+}$-DTZ complexes).
In a solution containing $10 \mathrm{ppm}$ of $\mathrm{Hg}^{2+}$ (Fig. 3d), both the CA-AA-DTZ and CA-FA-DTZ sensor strips displayed full color changes from green to orange and light magenta, respectively. These colors fall in the hot-color region and were found to show an increase in the data plot intensity at the $R$-axis. Interestingly, the color intensity plot at the $G$-axis (green color) for the CA-FADTZ membrane was found to be shifted to the left. The decrease in green color intensity (original color for the CA-FA-DTZ membrane) was due to the formation of $\mathrm{Hg}^{2+}$-DTZ complexes when the higher concentrations of $\mathrm{Hg}^{2+}$ (5 ppm and $\left.10 \mathrm{ppm}\right)$ were tested. All the detection sites of the CA-FA-DTZ membrane were occupied to execute the complete color change (with no green color observed), and hence, a lower value of the color intensity was observed at the $G$-axis. However, the green background was still visible at the $G$-axis for the CA-AA-DTZ membrane, even at $5 \mathrm{ppm}$ and $10 \mathrm{ppm}$ of the $\mathrm{Hg}^{2+}$ solution. The color intensity plot at the $G$-axis was not reduced as expected. This might be due to the entrapment of DTZ inside the larger pore structure (Fig. 3a) of the CA-AA-DTZ membrane, which reduces the efficiency of the color change during $\mathrm{Hg}^{2+}$ detection.

Despite the fact that there was some inconsistency of color distribution on the membrane strip at low $\mathrm{Hg}^{2+}$ concentration, i.e., some overlapping color intensity plots at $3 \mathrm{ppm}$ for the CAAA-DTZ membrane, the DTZ-CA sensor strips demonstrated higher hot-color intensity with an increase in the metal concentration. In this work, the lowest concentration of $\mathrm{Hg}^{2+}$ ions used for the detection was at $3 \mathrm{ppm}$. However, the current study was not specifically designed to observe the amount of the detected metal, but focused on the improvement of metal detection by manipulating the membrane physical structure and chemical properties.

The DTZ loading capacity is closely related to the physical microstructure of a membrane. Therefore, an interconnected porous substrate was needed (in the present work the porous CA membrane was used) to accommodate the immobilization of the optical ligand, DTZ, onto the membrane surface, as to interact with $\mathrm{Hg}^{2+}$ in the testing solution. A porous membrane is critical for the rapid exposure of dithizone to the testing samples containing potential analytes. Indeed, a sensor is often better served by the use of a highly-porous membrane as the detection platform. As for the membrane physical structure, the miscibility/solubility parameter between the solvent and nonsolvent during the phase inversion procedure in membrane formation will strongly affect the membrane porosity. In this study, deionized water (DI) was utilized as the non-solvent to induce membrane porosity and formic acid (FA) acted as the solvent to dissolve the CA polymer. The ratio of FA to DI was adjusted based on a constant CA polymer weight of $17 \mathrm{wt} \%$ and the SEM micrographs of the synthesized membranes are presented in Fig. 4.

The CA-FA membrane (Fig. 4a and d) exhibits a dense-skin layer with a thickness of $7.25 \mu \mathrm{m}$ and also possesses a sponge-like porous substructure beneath this dense skin. The membrane comprises a larger surface area with its own characteristic smaller pores. Indeed, the formation of the dense-skin layer was attributed to the differences in the solubility 


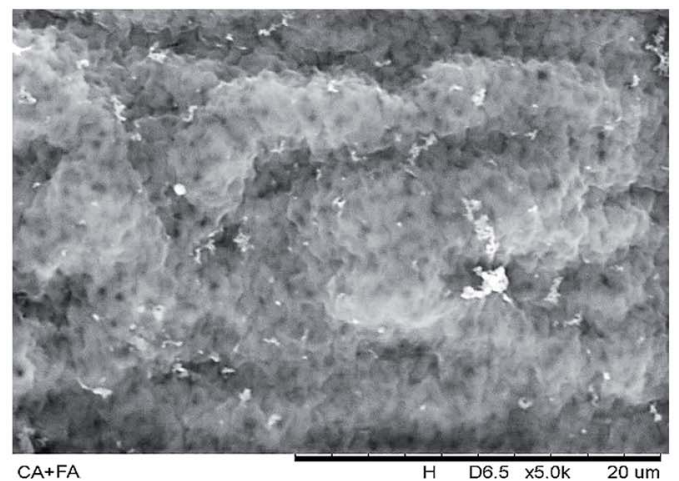

(a)

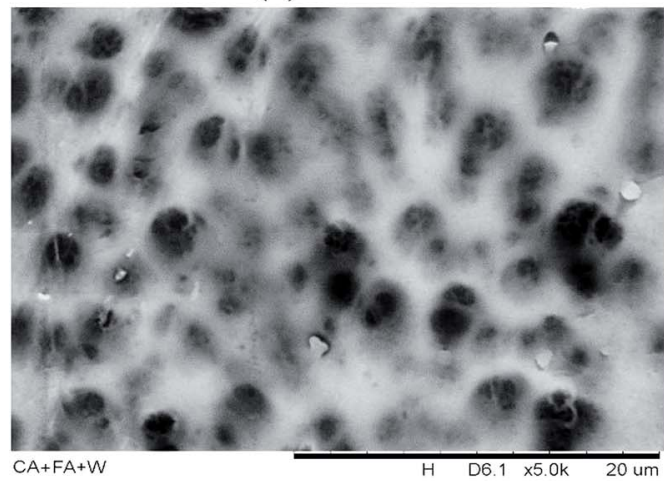

(b)

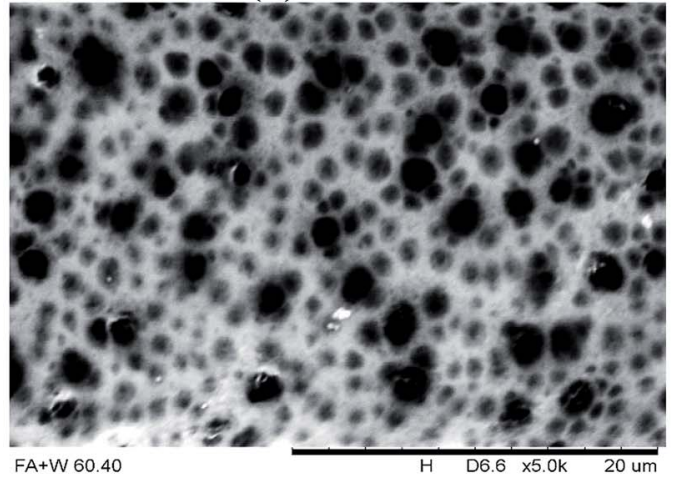

(c)

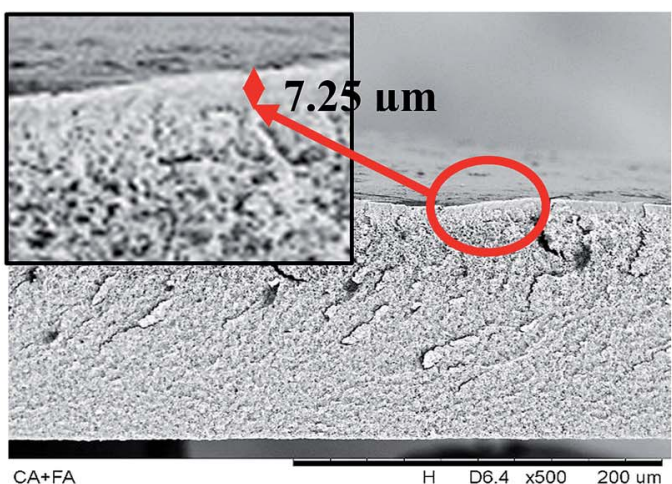

(d)

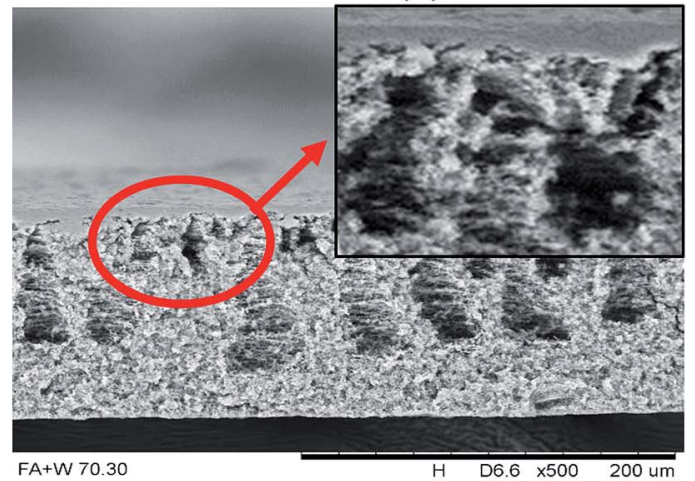

(e)

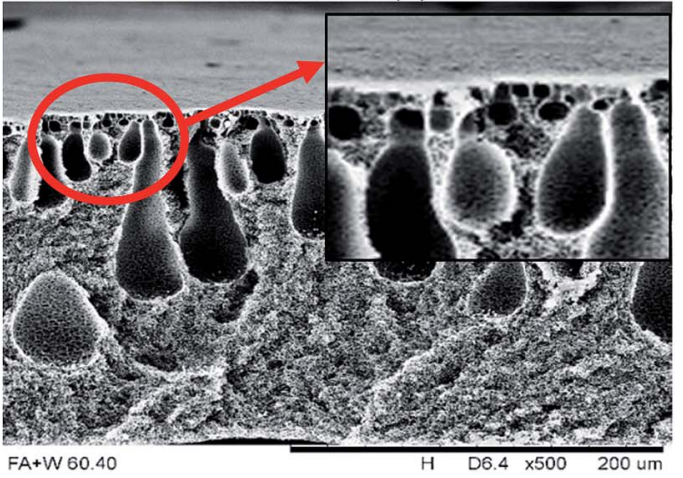

(f)

Fig. 4 SEM surface and cross-sections for ( $a$ and d) CA-FA, (b and e) CA-FA:30DI and (c and f) CA-FA:40DI membranes.

parameters for FA solvent in the casting dope and water in the coagulant bath during membrane solidification. The CA polymer, FA solvent, and water have Hansen solubility indices of $25.1 \mathrm{MPa}^{1 / 2}, 27.6 \mathrm{MPa}^{1 / 2}$ and $47.9 \mathrm{MPa}^{1 / 2}$, respectively. ${ }^{4-50}$ The high solubility differences in FA $\left(27.6 \mathrm{MPa}^{1 / 2}\right)$ and water $(47.9$ $\mathrm{MPa}^{1 / 2}$ ) caused delayed demixing during phase inversion, which is often associated with the formation of a dense skin top-layer on the membrane-air interface.

In contrast, when the non-solvent (deionized water, DI) was added into the casting dope, the CA FA:30DI membrane displays a porous surface (Fig. 4b) with a sponge-like shape around the finger-like structure in the cross-sectional image
(Fig. 4e). It can be assured that this type of membrane consists of larger pores and has higher porosity, compared to the CA-FA membrane. As noted above, the differences in the solubility parameters for the solvent and non-solvent affect the membrane morphology. The highly porous and finger-like structures found in Fig. $4 \mathrm{~b}$ and e may due to the mixing of the solubility properties of FA and DI in the casting dope. This creates a smaller difference between the solubility properties of the casting dope (mixing of FA and water) and those of the coagulation bath (water), thus enhancing the miscibility of the casting dope (FA-DI) with the non-solvent (DI water) during solvent exchange. Furthermore, the addition of a small amount 
of water in the casting dope will reduce the thermodynamic miscibility of the dope and result in faster precipitation during phase inversion. ${ }^{51}$ The thermodynamic instability of the casted film will enhance the diffusion of solvent into the coagulation bath $^{52}$ to form a solidified membrane.

As more DI water was added to the casting dope, the CAFA:40DI membrane demonstrated an even more porous structure (Fig. 4c). As is also apparent from Fig. 4f, finger-like structures were formed with larger voids compared to those of the CA-FA:30DI membrane (Fig. 4e). This proves that the addition of non-solvent (DI water) in the polymer dope is capable of increasing the membrane porosity.$^{53}$ Indeed, a membrane with high porosity has the advantage of facilitating the overall immobilization process, where the diffusion resistance of DTZ attached onto the porous matrix is lower, and there are plenty of voids. $^{27,42}$ However, further increasing the amount of water will cause the membrane to lose its mechanical properties due to the formation of macrovoids or over-sized finger-like structures. Furthermore, a highly porous membrane with larger pores tends to have relatively lower surface area availability, subsequently decreasing the total membrane surface area necessary for DTZ immobilization to take place.

Fig. 5 illustrates the effect of non-solvent composition on membrane porosity by showing the specific pore size and the pore diameter distribution. The pore size distribution was measured based on approximately 60 pores for each membrane, to increase the accuracy of the pore distribution trend. As seen in Fig. 5, the CA-FA membrane (Fig. 5a) appears to have the smallest pore size with a mean diameter of $0.319 \pm 0.149 \mu \mathrm{m}$. Meanwhile, with increasing DI content in the casting dope, the CA-FA:30DI membrane (Fig. 5b) and CA-FA:40DI membrane (Fig. 5c) have mean diameters of $0.698 \pm 0.217 \mu \mathrm{m}$ and $1.297 \pm$ $0.398 \mu \mathrm{m}$, respectively. These results are consistent with the variation in membrane structure indicated in Fig. 4, confirming that the addition of the higher amount of non-solvent in the dope solution leads to the formation of the larger membrane pores. ${ }^{53}$ Again, this confirms the ability of water to act as an effective pore former that increases the porosity of the membrane and leads to larger void structures.

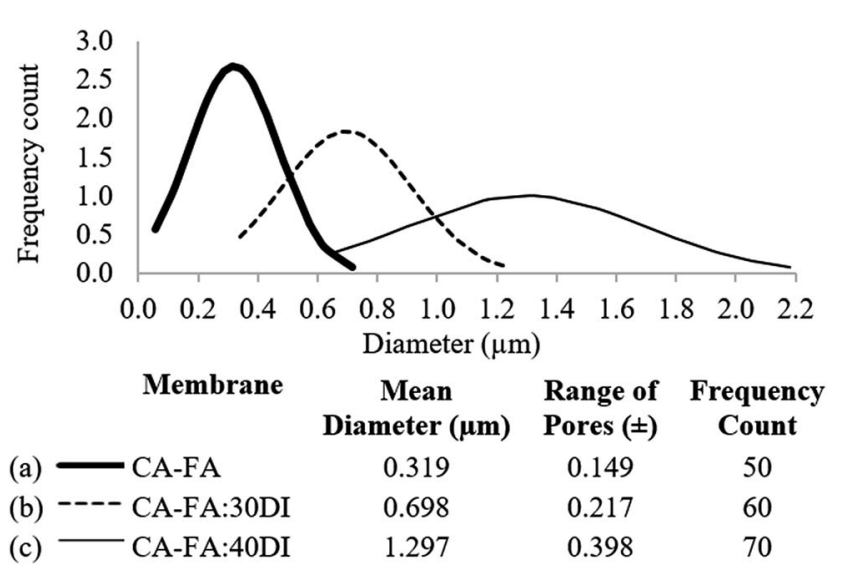

Fig. 5 Pore diameter distribution for (a) CA-FA, (b) CA-FA:30DI and (c) CA-FA:40DI membranes.
The increase in the membrane porosity was expected to give a different type of color assessment due to the varying level of DTZ immobilization on the membrane surface. The performances of the DTZ-immobilized CA-FA, CA-FA:30DI and CAFA:40DI membranes for the detection of $\mathrm{Hg}^{2+}$ (3-10 ppm) were assessed, and the results are displayed in Fig. 6 .

Contrary to expectations, there were no significant differences in the RGB values (green color) of all control membranes prior to $\mathrm{Hg}^{2+}$ detection. In other words, the immobilization level of DTZ on the membrane surfaces was the same, regardless of the variations in membrane structure. However, when the membranes were tested with $3 \mathrm{ppm} \mathrm{Hg}^{2+}$ solution, differences in the RGB values were observed, as highlighted in Fig. 6 and 7. Not all of the surface area of the CA-FA-DTZ sensor strip changed from green to greenish-orange color; thus, the distribution of the RGB values was close to that of the control membrane (Fig. 6a). Meanwhile, major color changes from green to light magenta and greenish-orange for the CA-FA:30DIDTZ (Fig. 6b) and CA-FA:40DI-DTZ (Fig. 6c) membranes, respectively, were identified.

In Fig. 6b, the RGB values for the CA-FA:30DI-DTZ membrane sensor tested with $3 \mathrm{ppm}$ of $\mathrm{Hg}^{2+}$ are far from those of the control membrane and are close to the hot-color region, relative to that of the CA-FA:40DI-DTZ (Fig. 6c) and CA-FA-DTZ (Fig. 6a) membranes. As mentioned earlier, the greater the distance of color distribution from the control membrane, the better the sensitivity of the membrane sensor to detect $\mathrm{Hg}^{2+}$. Fig. 7 further analyzes the color intensity at the $R$ axis for the membrane sensors tested in $3 \mathrm{ppm} \mathrm{Hg}^{2+}$. The $R$-axis was chosen because it is the hot-color region that represents the color changes due to the formation of $\mathrm{Hg}^{2+}$-DTZ complexes. As expected, the CA-FA:30DI-DTZ membrane demonstrated the highest difference in $R$-value from its control membrane, i.e. 117 in comparison to CA-FA-DTZ (63) and CA-FA:30DI-DTZ (93). As discussed earlier, the CA-FA:30DI-DTZ membrane comprises a porous surface with finger-like structures, and it is thus easier for DTZ molecules to be immobilized on the membrane surface, further increasing the detection efficiency. Logically, the CAFA:40DI-DTZ membrane, which has the highest porosity, should show better detection. However, the membrane was found to have over-sized pores, i.e. $1.3 \mu \mathrm{m}$, which is almost twofold $(0.7 \mu \mathrm{m})$ and four-fold $(0.3 \mu \mathrm{m})$ that of the CA-FA:30DI-DTZ and CA-FA-DTZ membranes, respectively. The total surface area of this membrane was found to be the lowest and thus, the membrane possesses fewer binding sites to accommodate DTZ molecules, which is further demonstrated by the lower efficiency of the sensor.

It was hypothesized that the sensor would respond differently (different RGB values) when a higher amount of DI (nonsolvent) is added into the casting dope, due to the different membrane microstructures that affect DTZ immobilization. However, a closer inspection revealed that there was only a small difference in RGB values for the CA-FA:30DI-DTZ and

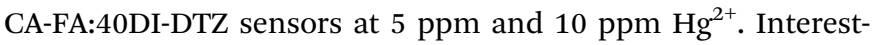
ingly, both CA-FA:30DI-DTZ and CA-FA:40DI-DTZ demonstrated similar color intensity plots, as shown in Fig. 6b and c, respectively. Both types of sensors (Fig. 6b and c) exhibited 


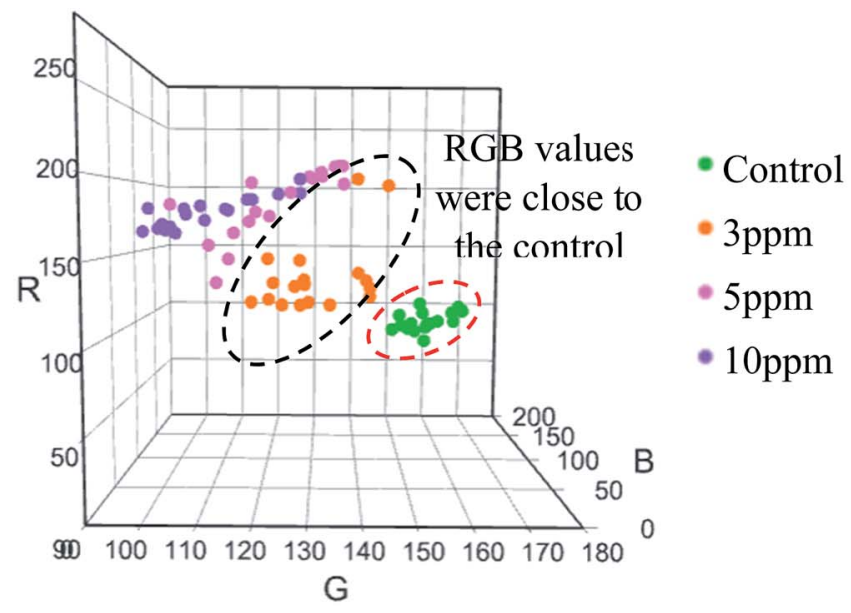

(a)

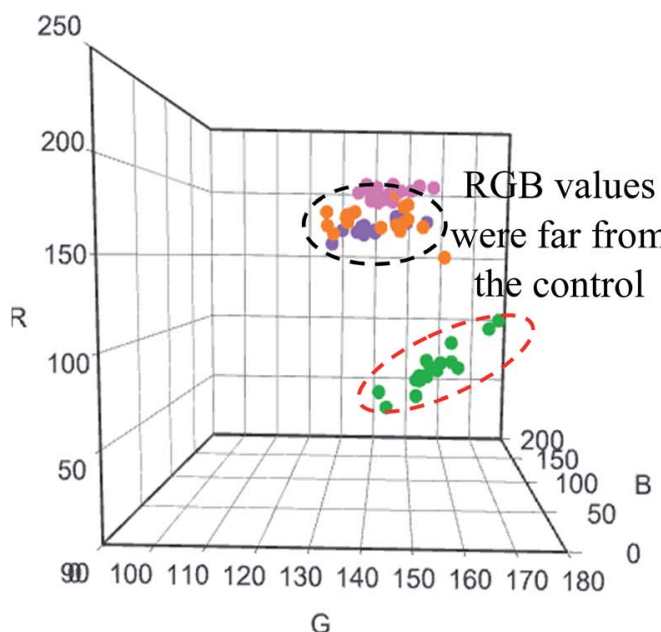

(b)

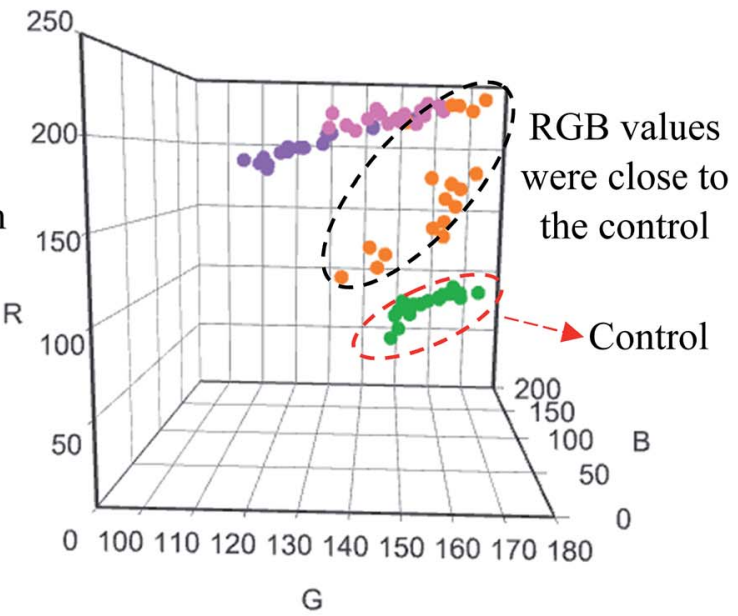

(c)

Fig. 6 Performances of the DTZ-colorimetric sensors: (a) CA-FA, (b) CA-FA:30DI and (c) CA-FA:40DI, for the detection of Hg ${ }^{2+}$.

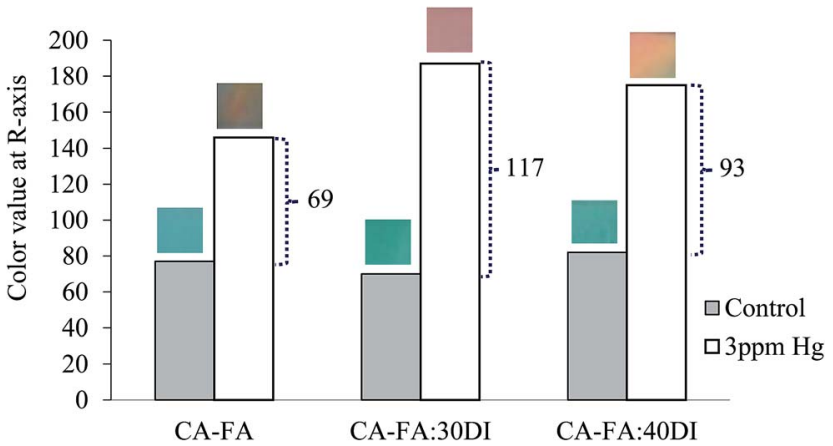

Fig. 7 Color value at $R$-axis for DTZ-colorimetric sensor: (a) CA-FA, (b) CA-FA:30DI and (c) CA-FA:40DI tested with $3 \mathrm{ppm} \mathrm{Hg}^{2+}$ solution.

overlapping RGB data when tested with $5 \mathrm{ppm}$ and $10 \mathrm{ppm}$ $\mathrm{Hg}^{2+}$. This might be due to color saturation effects. Despite this, the above results still confirm that an appropriate amount of DI water in the casting dope does play a prominent role in improving the sensitivity of the membrane sensor to detect $\mathrm{Hg}^{2+}$. In this work, CA-FA:30DI-DTZ performed best, and was found to have a detection sensitivity of $3 \mathrm{ppm}$, which is preferable for metal sensing applications.

\section{Conclusions}

Taken together, these findings highlight the role of membrane structure in the successful immobilization of dithizone (DTZ). The CA membrane-based sensors with DI water added as a nonsolvent (CA-FA:30DI-DTZ and CA-FA:40DI-DTZ) demonstrate higher membrane porosity, which improves the detection sensitivity for $\mathrm{Hg}^{2+}$. All of the prepared membrane sensors demonstrate obvious color changes at $5 \mathrm{ppm}$. Nevertheless, membrane sensors with high sensitivity (ability to detect lower $\mathrm{Hg}^{2+}$ concentration) are preferable for metal sensing applications. In this work, the CA-FA:30DI-DTZ membrane sensor showed better potential to detect the lower concentration of $\mathrm{Hg}^{2+}$ (3 ppm). 


\section{Conflicts of interest}

There are no conflicts of interest to declare.

\section{Acknowledgements}

The authors gratefully acknowledge the financial support from MOSTI EScience Fund (305/PJKIMIA/6013393). N. A. Azmi is financially assisted by the Ministry of Higher Education (MOHE).

\section{References}

1 M. Barakat, Arabian J. Chem., 2011, 4, 361-377.

2 M. Castro-González and M. Méndez-Armenta, Environ. Toxicol. Pharmacol., 2008, 26, 263-271.

3 S. Majumder, G. Gangadhar, S. Raghuvanshi and S. Gupta, J. Water Process Eng., 2015, 6, 136-143.

4 P. B. Tchounwou, C. G. Yedjou, A. K. Patlolla and D. J. Sutton, Heavy Metal Toxicity and the Environment, Springer Basel, Basel, 2012.

5 H. Jaman, D. Chakraborty and P. Saha, Clean: Soil, Air, Water, 2009, 37, 704-711.

6 S. M. Husnain, J.-H. Kim, C.-S. Lee, Y.-Y. Chang, W. Um and Y.-S. Chang, RSC Adv., 2016, 6, 35825-35832.

7 A. Rezaee, J. Derayat, S. Mortazavi, Y. Yamini and M. Jafarzadeh, Am. J. Environ. Sci., 2005, 1, 102-105.

8 B. V. Tangahu, S. R. Sheikh Abdullah, H. Basri, M. Idris, N. Anuar and M. Mukhlisin, Int. J. Chem. Eng., 2011, 2011, 31. 9 R. Sitko, M. Musielak, B. Zawisza, E. Talik and A. Gagor, RSC Adv., 2016, 6, 96595-96605.

10 D. P. Torres, M. B. Martins-Teixeira, S. Cadore and H. M. Queiroz, J. Environ. Sci. Health, Part B, 2015, 50, 514522.

11 J. Fan, Y. Qin, C. Ye, P. Peng and C. Wu, J. Hazard. Mater., 2008, 150, 343-350.

12 M. Fayazi, M. A. Taher, D. Afzali and A. Mostafavi, Sens. Actuators, B, 2016, 228, 1-9.

13 E. A. Osipova, V. E. Sladkov, A. I. Kamenev, V. M. Shkinev and K. E. Geckeler, Anal. Chim. Acta, 2000, 404, 231-240.

14 Y. Zhang, G.-M. Zeng, L. Tang, Y.-P. Li, Z.-M. Chen and G.-H. Huang, RSC Adv., 2014, 4, 18485-18492.

15 M. R. Mahmoudian, W. J. Basirun and Y. Alias, RSC Adv., 2016, 6, 36459-36466.

16 L. Zhang, Y.-H. Zhao and R. Bai, J. Membr. Sci., 2011, 379, 6979.

17 J. S. Wilson, Sensor technology handbook, Elsevier, Burlington, 2004.

18 B. A. Garcia Grajeda, S. G. Soto Acosta, S. A. Aguila, H. P. Guevara, M. E. Diaz-Garcia, A. C. Enriquez and J. J. Campos-Gaxiola, RSC Adv., 2017, 7, 31611-31618.

19 Y.-R. Kim, R. K. Mahajan, J. S. Kim and H. Kim, ACS Appl. Mater. Interfaces, 2010, 2, 292-295.

20 M. Anju, T. Divya, M. P. Nikhila, T. V. A. Kusumam, A. K. Akhila, V. A. Ansi and N. K. Renuka, RSC Adv., 2016, 6, 109506-109513.
21 S. Kraithong, P. Damrongsak, K. Suwatpipat, J. Sirirak, P. Swanglap and N. Wanichacheva, RSC Adv., 2016, 6, 10401-10411.

22 K. T. Kim, H. S. Jung, J. Ahn, Y. Choi, J. H. Jung and J. Park, RSC Adv., 2016, 6, 79405-79409.

23 J. H. Kim, H. J. Kim, S. H. Kim, J. H. Lee, J. H. Do, H.-J. Kim, J. H. Lee and J. S. Kim, Tetrahedron Lett., 2009, 50, 59585961.

24 J. F. Zhang, C. S. Lim, B. R. Cho and J. S. Kim, Talanta, 2010, 83, 658-662.

25 T. Balaji, S. A. El-Safty, H. Matsunaga, T. Hanaoka and F. Mizukami, Angew. Chem., 2006, 118, 7360-7366.

26 I. Oehme and O. S. Wolfbeis, Microchim. Acta, 1997, 126, 177-192.

27 A. Safavi and M. Bagheri, Sens. Actuators, B, 2004, 99, 608-612. 28 M. Sun, B. Ding and J. Yu, RSC Adv., 2012, 2, 1373-1378.

29 I. C. Santos, R. B. Mesquita and A. O. Rangel, Talanta, 2015, 143, 359-365.

30 Y. Takahashi, S. Danwittayakul and T. M. Suzuki, Analyst, 2009, 134, 1380-1385.

31 L. Li, L. Liao, Y. Ding and H. Zeng, RSC Adv., 2017, 7, 1036110368.

32 M. Anari-Anaraki and A. Nezamzadeh-Ejhieh, J. Colloid Interface Sci., 2015, 440, 272-281.

33 K. Zargoosh and F. F. Babadi, Spectrochim. Acta, Part A, 2015, 137, 105-110.

34 B. K. Momidi, V. Tekuri and D. R. Trivedi, Spectrochim. Acta, Part A, 2017, 180, 175-182.

35 N. Azmi and S. Low, J. Water Process Eng., 2017, 15, 37-42.

36 M. K. LaGasse, J. M. Rankin, J. R. Askim and K. S. Suslick, Sens. Actuators, B, 2014, 197, 116-122.

37 Z. Xu, P. Deng, J. Li and S. Tang, Sens. Actuators, B, 2018, 255, 2095-2104.

38 S. H. Lim, J. W. Kemling, L. Feng and K. S. Suslick, Analyst, 2009, 134, 2453-2457.

39 M. Hong, X. Lu, Y. Chen and D. Xu, Sens. Actuators, B, 2016, 232, 28-36.

40 R. Zare-Dorabei, R. Rahimi, A. Koohi and S. Zargari, RSC Adv., 2015, 5, 93310-93317.

41 J. W. Kemling, A. J. Qavi, R. C. Bailey and K. S. Suslick, J. Phys. Chem. Lett., 2011, 2, 2934-2944.

42 Z. Zhang, J. Gao, W. Zhang and Z. Ren, Sep. Sci. Technol., 2006, 41, 3245-3263.

43 Z. A. Jawad, A. L. Ahmad, S. C. Low and S. Zein, Curr. Nanosci., 2015, 11, 69-79.

44 C. Yin, J. Iqbal, H. Hu, B. Liu, L. Zhang, B. Zhu and Y. Du, J. Hazard. Mater., 2012, 233, 207-212.

45 S. Danwittayakul, Y. Takahashi, T. Suzuki and A. Thanaboonsombut, J. Met., Mater. Miner., 2008, 18, 37-40.

46 B. Kumar, H. B. Singh, M. Katyal and R. L. Sharma, Microchim. Acta, 1991, 105, 79-87.

47 T. J. Dickerson, N. N. Reed, J. J. LaClair and K. D. Janda, J. Am. Chem. Soc., 2004, 126, 16582-16586.

48 C. M. Hansen, Hansen solubility parameters: a user's handbook, CRC press, 2007.

49 R. B. Romero, C. A. P. Leite and M. d. C. Gonçalves, Polymer, 2009, 50, 161-170. 
50 Z. A. Jawad, A. L. Ahmad, S. C. Low, T. L. Chew and S. H. S. Zein, J. Membr. Sci., 2015, 476, 590-601.

51 P. van de Witte, P. J. Dijkstra, J. W. A. van den Berg and J. Feijen, J. Membr. Sci., 1996, 117, 1-31.
52 J. Mulder, Basic Principles of Membrane Technology, Springer Science \& Business Media, Netherlands, 2012.

53 M. Z. Yunus, Z. Harun, H. Basri and A. F. Ismail, Adv. Mater. Res., 2012, 488-489, 46-50. 